\title{
Impact of Satellite Elevation Mask in GPS+Galileo RTK Positioning
}

\author{
J. Manuel Castro-Arvizu*, Daniel Medina*, Ralf Ziebold* \\ * Institute of Communications and Navigation, German Aerospace Center (DLR), Germany
}

\begin{abstract}
Global Navigation Satellite Systems (GNSS) have become the keystone and main information supplier for Positioning, Navigation and Timing (PNT) data. While providing an adequate open sky performance, the accuracy of standard code-based GNSS techniques is insufficient for applications requiring precise navigation. Additionally, GNSS positioning algorithms performance can be easily disturbed in signal-degraded environments due to space weather events, obstacles, urban areas, bridges, limited open sky view and/or low-elevation multipath effects. Hence, this study gives a comparative of the performance assessment for different high elevation masks of a multi-frequency multi-GNSS RTK method in a loose combination (a pivot satellite is chosen for each constellation and frequency) to avoid the case of the lack of coinciding frequencies and based in the code and carrier phase measurements of the integration of multiple GNSS constellations. The analysis of the Ambiguity Dilution of Precision (ADOP) and the number of fixed ratio epochs in both static and dynamic real scenarios, demonstrates that the dual frequency L1/E1+L5/E5a GPS+Galileo RTK positioning solution approach presented in this study has a good performance in terms of reliability, positioning accuracy and availability in comparative with a GPSonly RTK algorithm when high elevation mask values are used.
\end{abstract}

\section{INTRODUCTION}

During recent years, the data fusion of multiple GNSS systems gives the possibility of having a better performance in Positioning, Velocity and Timing (PVT) solutions in terms of availability, accuracy and reliability in engineering and scientific applications [1-3]. Several GNSS-driven positioning solutions exist in literature and they are used depending of the data type, number of receivers, number of observation samples and user applications. This work is focused on Real Time Kinematic (RTK), a relative positioning procedure where the position of a receiver is determined with respect to a stationary base station of accurately known coordinates [4]. RTK uses double difference (DD) code and carrier phase measurements, eliminating atmospheric effects, clock offsets and both receiver/satellites biases obtaining a centimeter-level positioning accuracy.

The use of carrier phase observations is a main factor for precise navigation, since their noise is two orders of magnitude lower than code observations. However, carrier observations are ambiguous by an unknown number of integer ambiguities. The process of determining the integer ambiguities, denoted as Integer Ambiguity Resolution (IAR) [5], grants the estimation of precise positioning. The goal of IAR is to use the integer ambiguity constraints for the improvement of the navigation solution. The RTK functional model is generally expressed as a Least-squares (LS) adjustment, for which a closed-form solution does not exist. Thus, its minimization is resolved applying a threesteps decomposition. The first step, "float estimation", constitutes a LS procedure where the integer constraint on the ambiguities is discarded. Then, the IAR estimates the integer ambiguities based on the float solution. This stage is commonly solved applying the Least-squares AMBiguity Decorrelation Adjustment (LAMBDA) method $[6,7]$. Finally, "solution fixing" realizes the enhancement on the position solution based on the estimated integer ambiguities.

GNSS-based positioning performance can be easily deteriorated during the navigation in urban scenarios, due to signal reflection and occlusion, limited open sky view and/or low-elevation multipath effects. Thus, the use of low-elevation satellites is limited in such signal-degraded scenarios. In the opposite side, the use of a high elevation mask carries a significant effect, since the geometry can deteriorate and the number of observations not be sufficient. These features drive to a poor performing IAR and thus a insufficiently positioning accuracy. However, when data fusion of multiple GNSS is used, the improvement of the IAR increases even when high elevation masks of satellites ares considered $[2,8,9]$. 
Hence, this study provides a performance assessment for different cut-off masks and a dual-frequency L1/L5 RTK algorithm, configured in a loose combination to avoid the case of the lack of coinciding frequencies and based in the code and carrier phase measurements of the integration of multiple GNSS constellations. The comparative between combined GPS-Galileo and GPS-only performances is verified also.

The importance of a good IAR results in a good positioning performance. One technique to measure the quality of the IAR is the Ambiguity Dilution of Precision (ADOP) [10,11]. ADOP gives a measure of the strength of the IAR and the geometry of the ambiguities. ADOP has been studied in literature for GPS-only performance [12, 13] and also for multiple GNSS systems $[2,11,14]$ showing that when number of satellites increases, the ADOP has better performance of ADOP assuring a prominent accuracy in terms of positioning.

The remainder of the paper is organized as follows. The description of a GPS+Galileo measurement model in a loose combination is given in Section Combined GPS+Galileo measurement model. The state space model and technical description of the combined GPS +Galileo RTK positioning algorithm proposed in this work is depicted in Section State Space Model for Float Solution. Section Fixed Solution: Ambiguity Resolution describes the technical solution for the IAR and ADOP description. Section Ambiguity Resolution Analysis and Results illustrates results with real data and Section Conclusions concludes the paper with final remarks.

\section{COMBINED GPS+GALILEO MEASUREMENT MODEL}

The main feature of RTK positioning technique is the simplification in the DD resolution of the phase integer ambiguities. Figure 1 depicts a basic diagram of RTK technique. For every system there is a base satellite (Loose combination). In the other hand where a base satellite is used for multiple systems is called tight combination permitting double differentiating across different systems. This work is centered in a loose RTK implementation.

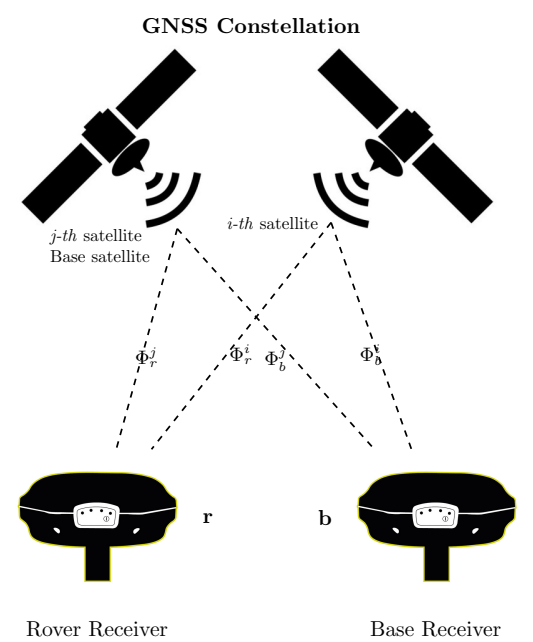

Figure 1: RTK Diagram where the base satellite is the pivot of one single system.

The between receiver Single Difference (SD) observation is expressed as:

$$
\begin{aligned}
\Delta \phi_{r-b}^{i} & =\Delta \rho_{r-b}^{i}+c \delta t_{r-b}+\lambda N_{r-b}^{i}+\epsilon_{r-b}^{i}, \\
\Delta C_{r-b}^{i} & =\Delta \rho_{r-b}^{i}+c \Delta t_{r-b}+\xi_{r-b}^{i},
\end{aligned}
$$

where $\delta t_{r-b}$ is the clock bias for rover $(r)$ and base $(b)$ stations; $c$ is the speed light; $\phi$ is the phase and $C$ and code measurements; $\rho$ is the range between the satellite and receiver; $N$ is the number of integer ambiguities; $\lambda$ denotes the wavelength at the corresponding frequency; $\epsilon$ and $\xi$ are the measurement noise for phase and code observations respectively. The subprefix $r-b$ means the difference between rover and base station according to the $i^{t h}$ satellite.

The respective DD equations where the receiver biases can be eliminated from Equations 1 and 2 according to 
Figure 1, finally can be expressed as:

$$
\begin{aligned}
\nabla \Delta \phi_{r-b}^{i-j} & =\nabla \Delta \rho_{r-b}^{i-j}+\lambda \nabla \Delta N_{r-b}^{i-j}+\epsilon_{r-b}^{i-j}, \\
\nabla \Delta C_{r-b}^{i-j} & =\nabla \Delta \rho_{r-b}^{i-j}+\xi_{r-b}^{i-j},
\end{aligned}
$$

where the subprefix $i-j$ means the DD of code and phase of the $i^{\text {th }}$ satellites according to the $j^{\text {th }}$ base satellite. Equations 3 and 4 are DD general expressions for one single-system only. However, for a a muti-GNSS case, several pair of these equations exist for every system. Thus this study is focused in a RTK position solution using a combined GPS + Galileo measurement set data, the general measurement subset vector DD observations for GPS-only (G) system is:

$$
\begin{aligned}
\nabla \Delta \boldsymbol{\phi}^{G} & =\nabla \Delta \boldsymbol{\rho}_{r-b}^{G}+\lambda \nabla \Delta \boldsymbol{N}_{r-b}^{G}+\boldsymbol{\epsilon}_{r-b}^{G}, \\
\nabla \Delta \boldsymbol{C}^{G} & =\nabla \Delta \boldsymbol{\rho}_{r-b}^{G}+\boldsymbol{\xi}_{r-b}^{G}
\end{aligned}
$$

Likewise for Galileo (E) system:

$$
\begin{aligned}
\nabla \Delta \boldsymbol{\phi}^{E} & =\nabla \Delta \boldsymbol{\rho}_{r-b}^{E}+\lambda \nabla \Delta \boldsymbol{N}_{r-b}^{E}+\boldsymbol{\epsilon}_{r-b}^{E}, \\
\nabla \Delta \boldsymbol{C}^{E} & =\nabla \Delta \boldsymbol{\rho}_{r-b}^{E}+\boldsymbol{\xi}_{r-b}^{E}
\end{aligned}
$$

So when in Equation 6 and 8 the base satellite (pivot) changes in a single epoch, the inter-system bias is still consistent and the continuous RTK positioning algorithm is still accomplished [15].

Two of the main features of a RTK algorithm is obtaining a Float Solution and a Fixed Solution. Float solution is referred as an Extended Kalman Filter $\mathbf{x}=\left[\mathbf{p}, \mathbf{v}, \boldsymbol{N}_{G}, \boldsymbol{N}_{E}\right]$, where $\mathbf{p}$ is the XYZ-position vector, $\mathbf{v}$ is the XYZvelocity vector; $\boldsymbol{N}_{G}$ is the SD float ambiguity vector of GPS and likewise $\boldsymbol{N}_{E}$ is the SD float ambiguity vector of Galileo.

\section{STATE SPACE MODEL FOR FLOAT SOLUTION}

The relation between code/phase measurements and position is defined as $\mathbf{y}_{k}=\left[\begin{array}{llll}\nabla \Delta \phi^{G} & \nabla \Delta \phi^{E} & \nabla \Delta C^{G} & \nabla \Delta C^{E}\end{array}\right]=$ $\mathbf{h}_{k}\left(\mathbf{x}_{k}\right)+\boldsymbol{\nu}_{k}$, where $\mathbf{y}_{k} \in \mathbb{R}^{2 n}$ is the observation vector and corresponds to the code and phase double differences; the observation error $\boldsymbol{\nu}_{k}$ is assumed to be zero-mean normal-distributed of covariance $\mathbf{R}_{k}$, and the nonlinear observation function, $\mathbf{h}_{k}\left(\mathbf{x}_{k}\right)$,

$$
\mathbf{h}_{k}=\left[\begin{array}{c}
\nabla \Delta \boldsymbol{\rho}_{r-b}^{G}+\lambda_{G} \boldsymbol{D} \cdot \boldsymbol{N}_{G} \\
\nabla \Delta \boldsymbol{\rho}_{r-b}^{E}+\lambda_{E} \boldsymbol{D} \cdot \boldsymbol{N}_{E} \\
\nabla \Delta \boldsymbol{\rho}_{r-b}^{G} \\
\nabla \Delta \boldsymbol{\rho}_{r-b}^{E}
\end{array}\right],
$$

and the corresponding Jacobian used to solve the nonlinear filtering problem into an EKF solution is given by derivatives $\frac{\delta \mathbf{h}_{k}}{\delta \mathbf{x}_{k}}$ is:

$$
\mathbf{H}_{k}=\left[\begin{array}{cccccc}
\frac{\delta \nabla \Delta \boldsymbol{\rho}_{r-b}^{G}}{\delta \mathbf{p}_{E}} & \mathbf{0} & \lambda_{G} \mathbf{D}^{G} & \mathbf{0} & \mathbf{0} & \mathbf{0} \\
\frac{\delta \nabla \Delta \boldsymbol{\rho}_{r-b}}{\delta \mathbf{p}_{b}} & \mathbf{0} & \mathbf{0} & \lambda_{E} \mathbf{D}^{E} & \mathbf{0} & \mathbf{0} \\
\frac{\delta \nabla \Delta \boldsymbol{\rho}_{r-b}^{G}}{\delta \mathbf{p}_{-b}} & \mathbf{0} & \mathbf{0} & \mathbf{0} & \mathbf{0} & \mathbf{0} \\
\frac{\delta \nabla \Delta \boldsymbol{\rho}_{r-b}^{E}}{\delta \mathbf{p}} & \mathbf{0} & \mathbf{0} & \mathbf{0} & \mathbf{0} & \mathbf{0}
\end{array}\right]
$$

Notice that the measurement Jacobian matrix in (10) is evaluated at the predicted state to obtain a linear formulation. In Equation 10, $\mathbf{D}^{G / E}$ is the DD model for either GPS or Galileo. If there are $m_{G}$ common GPS satellites, $m_{E}$ common Galileo satellites and the $m_{G}^{t h}$ and $m_{E}^{t h}$ are the base satellite for GPS and Galileo respectively, $\mathbf{D}^{G} \triangleq \mathbf{D}_{m_{G}-1 \times m_{G}}$ can be defined as $\mathbf{D}_{m_{G}-1 \times m_{G}}=\left[-\mathbf{e}_{m_{G}-1}, \mathbf{I}_{m_{G}-1 \times m_{G}-1}\right]$ for GPS system. Likewise $\mathbf{D}^{E} \triangleq$ 
$\mathbf{D}_{m_{E}-1 \times m_{E}}$ for Galileo system is expressed as $\mathbf{D}^{E} \triangleq \mathbf{D}_{m_{E}-1 \times m_{E}}[15,16]$. Finally, for a combined GPS + Galileo System, the $\mathbf{D}$ model is stated as:

$$
\mathbf{D}=\left[\begin{array}{cc}
\boldsymbol{I}_{2 \times 2} \otimes \mathbf{D}_{m_{G}-1 \times m_{G}} & \mathbf{0}_{m_{G}-1 \times m_{E}} \\
\mathbf{0}_{m_{E}-1 \times m_{G}} & \boldsymbol{I}_{2 \times 2} \otimes \mathbf{D}_{m_{E}-1 \times m_{E}}
\end{array}\right]
$$

where $\mathbf{e}_{m_{G / E}}$ is the $m_{G / E}^{t h}$ column vector full of elements of 1 . The full Kalman Filter algorithm can be written as follows:

Algorithm 1: EKF formulation for float solution

Require: $\hat{\mathbf{x}}_{0 \mid 0}, \mathbf{P}_{x, 0 \mid 0}, \mathbf{Q}_{k}$ and $\mathbf{R}_{k}, \forall k$

1: Set $k \Leftarrow 1$

2: Estimate the predicted state:

3: Estimate the predicted error covariance:

Time update (prediction)

$$
\begin{gathered}
\hat{\mathbf{x}}_{k \mid k-1}=\mathbf{F}_{x} \hat{\mathbf{x}}_{k-1 \mid k-1} . \\
\mathbf{P}_{x, k \mid k-1}=\mathbf{F}_{x} \mathbf{P}_{x, k-1 \mid k-1} \mathbf{F}_{x}^{\top}+\mathbf{Q}_{k} .
\end{gathered}
$$

4: Estimate the predicted measurement:

5: Estimate the innovation covariance matrix:

Measurement update (estimation)

6: Estimate the Kalman gain

7: Estimate the updated state

8: Estimate the corresponding error covariance:

$$
\begin{gathered}
\hat{\mathbf{y}}_{k \mid k-1}=\mathbf{h}_{k}\left(\hat{\mathbf{x}}_{k \mid k-1}\right) . \\
\mathbf{P}_{y, k \mid k-1}=\mathbf{H}_{k} \mathbf{P}_{x, k \mid k-1} \mathbf{H}_{k}^{\top}+\mathbf{R}_{k} . \\
\tilde{\mathbf{K}}_{k}=\mathbf{P}_{x, k \mid k-1} \mathbf{H}_{k}^{\top} \mathbf{P}_{y, k \mid k-1}^{-1} . \\
\hat{\mathbf{x}}_{k \mid k}=\hat{\mathbf{x}}_{k \mid k-1}+\tilde{\mathbf{K}}_{k}\left(\mathbf{y}_{k}-\hat{\mathbf{y}}_{k \mid k-1}\right) . \\
\mathbf{P}_{x, k \mid k}=\mathbf{P}_{x, k \mid k-1}-\tilde{\mathbf{K}}_{k} \mathbf{G}_{k} \mathbf{P}_{x, k \mid k-1} .
\end{gathered}
$$

9: Set $k \Leftarrow k+1$ and go to step 2 .

where $\boldsymbol{P}_{x, k}$ is the covariance matrix of the states at $k^{t h}$ epoch; $\boldsymbol{P}_{x, k \mid k-1}$ is the one-step estimation of $\boldsymbol{P}_{k} ; \tilde{\mathbf{K}}_{k}$ is the Kalman gain matrix; $F_{x}$ one-step state transition matrix; $\boldsymbol{Q}_{k}$ is the process noise covariance matrix and $\boldsymbol{R}_{k}$ is the covariance matrix for the DD expressed as:

$$
\boldsymbol{R}_{k}=\left[\begin{array}{cccc}
\boldsymbol{D} \cdot \boldsymbol{R}_{\phi}^{G} \cdot \boldsymbol{D} & \mathbf{0} & \mathbf{0} & \mathbf{0} \\
\mathbf{0} & \boldsymbol{D} \cdot \boldsymbol{R}_{\phi}^{E} \cdot \boldsymbol{D} & \mathbf{0} & \mathbf{0} \\
\mathbf{0} & \mathbf{0} & \boldsymbol{D} \cdot \boldsymbol{R}_{C}^{G} \cdot \boldsymbol{D} & \mathbf{0} \\
\mathbf{0} & \mathbf{0} & \mathbf{0} & \boldsymbol{D} \cdot \boldsymbol{R}_{C}^{E} \cdot \boldsymbol{D}
\end{array}\right]
$$

$\boldsymbol{R}_{C}$ and $\boldsymbol{R}_{\phi}$ are the code and carrier phase covariance measurement matrices respectively, whose characterization is addressed in $[17,18]$.

\section{FIXED SOLUTION: AMBIGUITY RESOLUTION}

The Least-squares Ambiguity Decorrelation Adjustment (LAMBDA) algorithm [6] is considered for the process of integer ambiguity resolution. After float solution implementation, from estimated state vector $\hat{\mathbf{x}}_{k \mid k}, \hat{\mathbf{N}}=\left[\begin{array}{ll}\hat{\boldsymbol{N}}_{G} & \hat{\mathbf{N}}_{E}\end{array}\right]^{\top}$ and its respective $\mathbf{P}_{N, k \mid k}$ taken from $\mathbf{P}_{x, k \mid k}$, are used as input for the Integer Least-square (ILS) LAMBDA estimator to obtain the integer DD ambiguity $N=\left[\begin{array}{ll}N_{G} & N_{E}\end{array}\right]^{\top}$. Then, the ratio-test upon the estimated ambiguities is realized to decide whether to accept or not the integer solution [19, 20]. In this case, a fixed threshold $R_{\text {threshold }}$ of $3.0[21]$ is considered:

$$
\tilde{N}=\arg \min _{N}\left[(N-\hat{N})^{\top} \cdot Q_{N}^{-1} \cdot(N-\hat{N})\right]
$$


where $\boldsymbol{Q}_{N}$ is the variance-covariance matrix of float ambiguities and the fix ratio computation is:

$$
R=\frac{\left(\tilde{\boldsymbol{N}}_{2}-\hat{\boldsymbol{N}}\right)^{\top} \cdot \boldsymbol{Q}_{N}^{-1} \cdot\left(\tilde{\boldsymbol{N}}_{2}-\hat{\mathbf{N}}\right)}{\left(\tilde{\boldsymbol{N}}_{1}-\hat{\mathbf{N}}\right)^{\top} \cdot \boldsymbol{Q}_{N}^{-1} \cdot\left(\tilde{\boldsymbol{N}}_{1}-\hat{\mathbf{N}}\right)}
$$

From equation 14 if $R>R_{\text {treshhold }}$, thus we have a fixed solution.

As result, and easy way to compute the model strength of ambiguity resolution process is the ambiguity dilution of precision (ADOP) $[11,12]$ which is given in cycles as:

$$
A D O P={\sqrt{\left|\boldsymbol{Q}_{N}\right|^{\frac{1}{n}}}}^{2}
$$

where $n$ is the size ambiguity vector and $|\cdot|$ denotes the determinant. A successful ambiguity resolution is considered when ADOP values is below a $A D O P_{\text {threshold }}$ of 0.12 cycles corresponding an to Ambiguity Success Rate (ASR) of $99.9 \%[11]$.

\section{AMBIGUITY RESOLUTION ANALYSIS AND RESULTS}

In this section, the study and analysis of the impact of the elevation mask of satellites in IAR for a combined GPS +Galileo RTK positioning solution are presented. As well, as the influence of a correct IAR in the position solution.

\section{Experimental Setup}

The validation of the combined GPS + Galileo RTK position approach presented in study was validated with real GNSS data recorded in a measurement campaign conducted in Koblenz, Germany in 2019 (DOYs 133 and 134). The data was collected on board of the vessel MS Bingen at $1 \mathrm{~Hz}$. The equipment consisted of a navXperience GNSS antenna connected to a geodetic Javad DELTA Receiver and a TRIMBLE receiver for RTCM3 messages corrections from the reference station with a ground truth known coordinates in Koblenz, Germany. Static data of DOY 133 (UTC 20:00 - 08:00) was used for the IAR analysis and the impact of the elevation mask of the combined GPS+Galileo RTK position solution approach studied in this work. The validation was made with the comparative with single frequency GPS-only System versus a Combined GPS+Galileo systems in dual frequency L1+L5 respectively. The analysis in the performance of the RTK positioning algorithm in loose combination approach proposed in this study was conducted for elevation masks of $10^{\circ}, 15^{\circ}, 25^{\circ}, 35^{\circ}$ and $45^{\circ}$ respectively using the GNSS measurement data of DOY 133. In order to track accurately the position of the vessel independently on the GNSS information, a geodetic total station was placed on the shores of the river (see Figure 10) to ensure the availability of the reference information even in areas where the GNSS performance was poor, as elaborated in [22].

\section{Results and Analysis}

Figure 2 gives a comparative of the number of satellites for both elevation masks of $10^{\circ}$ and $45^{\circ}$. Its corresponding ADOP values are shown in Figure 3 for a elevation mask of 10 and 45 respectively also. According to [7] and [11], for a combined GPS+Galileo System, it is possible obtaining an ADOP value below 0.12 cycles and consequently giving the certainty of a strong IAR a even for a high elevation mask in comparative with using a GPS-only system.

The percentage of ADOP values below 0.12 cycles for different elevation mask is plotted in Figure 4. This figure shows that, when the elevation mask increases, the percentage of ADOP values below of 0.12 cycles for a combined GPS + Galileo system is significant in comparative with using a GPS-only System. Thus, a combined system will give the certainty of an appropriate IAR and consequently an ASR of $99.9 \%$ even for high satellite elevation mask values.

An empirical analysis of the fulfilment of the IAR method is the Success Fix solution ratio (FR) defined as:

$$
F R=\frac{\text { Number of fixed solution }}{\text { Number of total epochs }} \times 100
$$



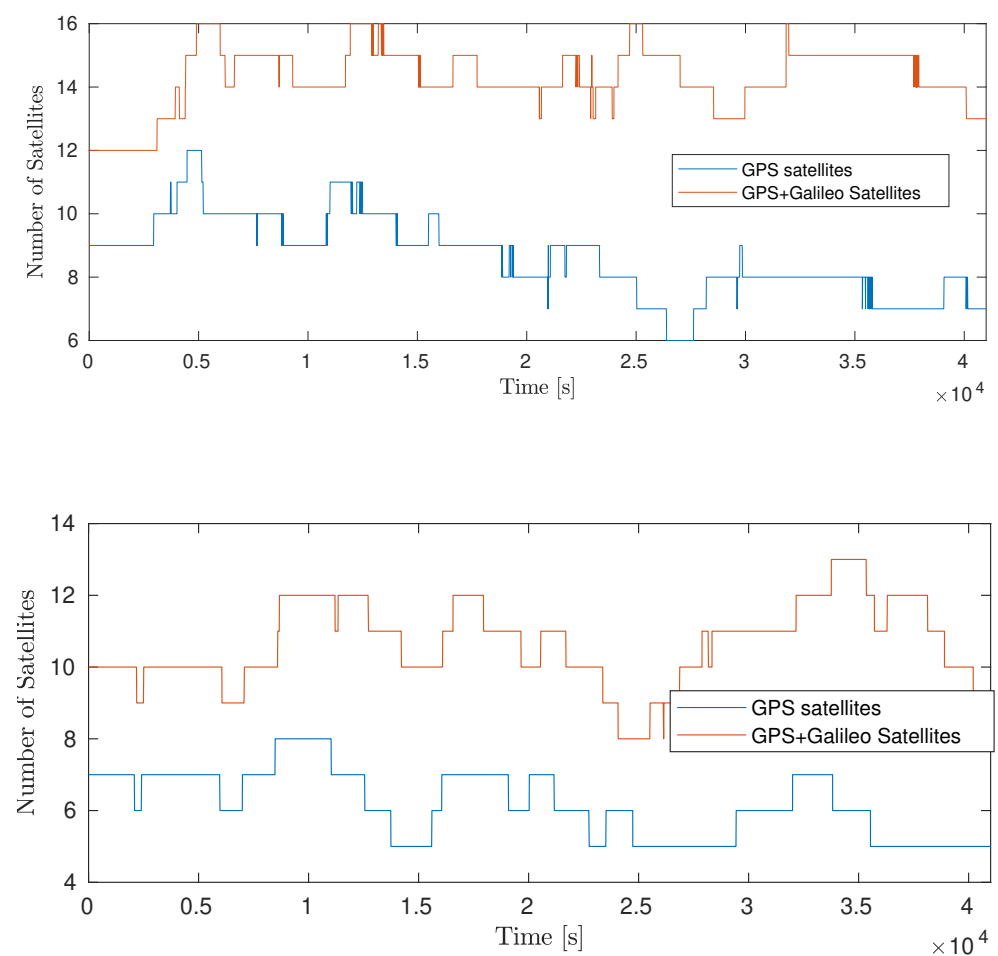

Figure 2: Number of GPS-only, GPS + Galileo satellites. Top Figure corresponds to the analysis of an elevation mask of $10^{\circ}$ and the bottom figure to the analysis of an elevation mask of $45^{\circ}$.
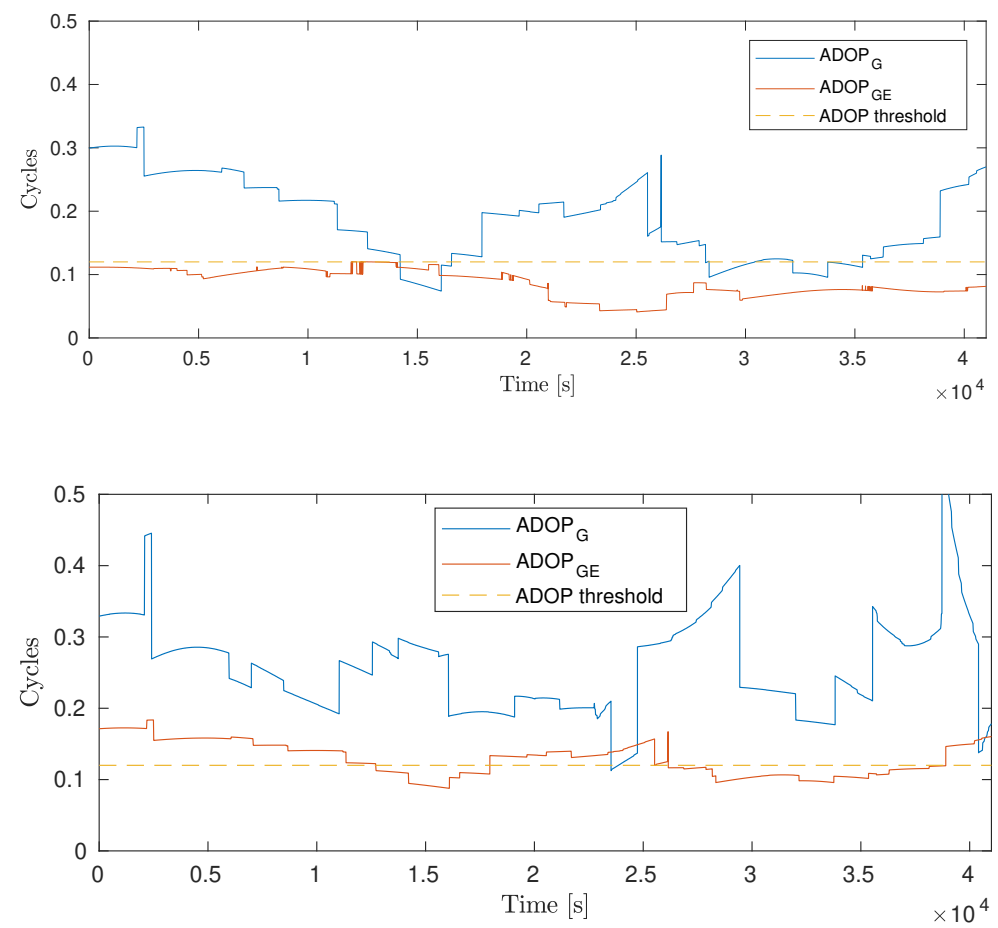

Figure 3: Top Figure corresponds to ADOP values when an elevation mask of $10^{\circ}$ is se. As comparison, the bottom Figure plots the ADOP values when a high elevation mask of $45^{\circ}$ is set. 


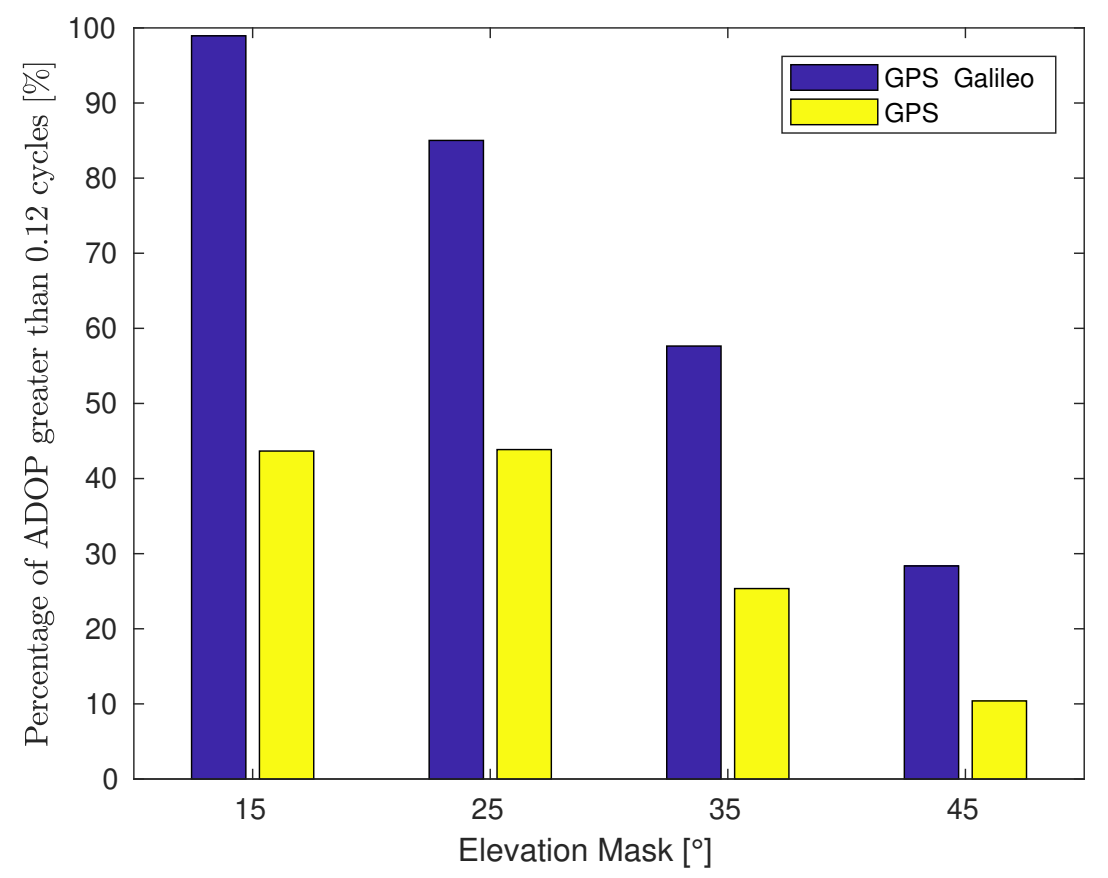

Figure 4: Percentage of ADOP values below 0.12 cycles for different elevation masks values.
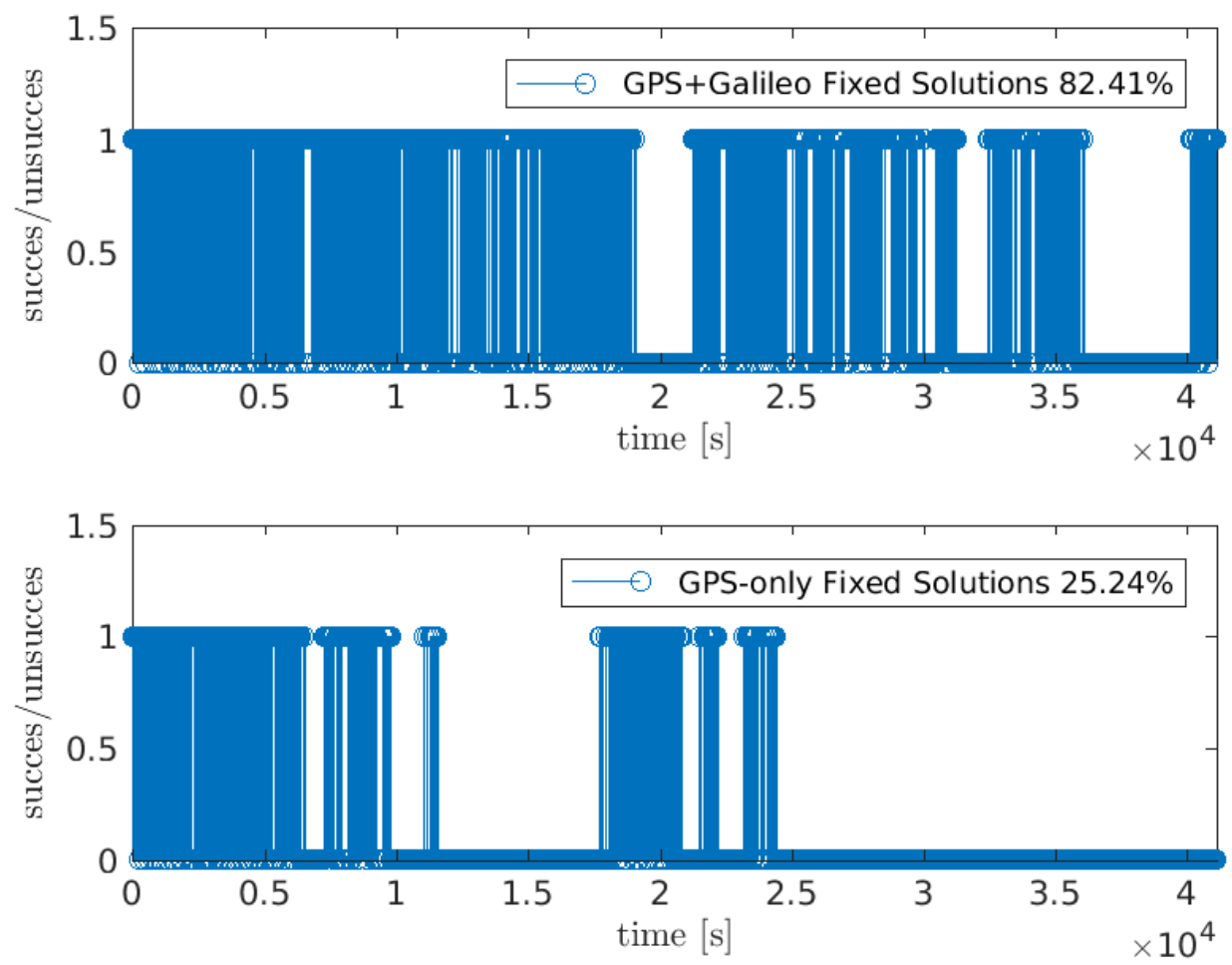

Figure 5: Success and unsuccessful fixed solutions with GPS-only and GPS + Galileo RTK positioning algorithm with elevation mask of $45^{\circ}$ 
The comparative between the solution using a combined system and a GPS only system is depicted in Figure 5. This Figure shows the percentage of Fixed solutions for a elevation mask of $45^{\circ}$. For a combined system, $82.41 \%$ percentage of epochs have a fixed solution in contrast with a GPS-only system with only $25.24 \%$ of fixed solutions.

Table 1 numerically depicts and resumes the values for different elevation masks and its impact in the mean ADOP, the percentage of ADOP values below 0.12 cycles and also the percentage of Success fixed solutions ratio. From this table the best approach for a good combination of ADOP values and percentage of fixed solution ratio is for a GPS + Galileo combined system when high satellite elevation mask are implicated.

\begin{tabular}{|c|c|c|c|c|c|c|c|c|c|}
\hline \multirow[b]{2}{*}{ System/Frequency } & \multicolumn{3}{|c|}{$10^{\circ}$} & \multicolumn{3}{|c|}{$15^{\circ}$} & \multicolumn{3}{|c|}{$25^{\circ}$} \\
\hline & $A D O P$ & $\begin{array}{l}A D O P \\
\leq \\
0.12(\%)\end{array}$ & $F R(\%)$ & $A D O P$ & $\begin{array}{l}A D O P \\
\leq \\
0.12(\%)\end{array}$ & $F R(\%)$ & $A D O P$ & $\begin{array}{l}A D O P \\
\leq \\
0.12(\%)\end{array}$ & $F R(\%)$ \\
\hline GPS L1 & 0.145 & 52.61 & 97.56 & 0.188 & 43.86 & 93.35 & 0.258 & 40.66 & 83.08 \\
\hline$G+E L 1 / L 5$ & 0.086 & 99.95 & 100 & 0.125 & 98.94 & 99.18 & 0.135 & 85.02 & 98.80 \\
\hline \multirow[b]{2}{*}{ System/Frequency } & \multicolumn{3}{|c|}{$35^{\circ}$} & \multicolumn{3}{|c|}{$45^{\circ}$} & & & \\
\hline & $A D O P$ & $\begin{array}{l}\% A D O I \\
\leq 0.12\end{array}$ & FR $(\%)$ & $A D O I$ & $\begin{array}{l}A D O P \\
\leq \\
0.12(\%)\end{array}$ & $F R(\%)$ & & & \\
\hline GPS L1 & 0.323 & 25.35 & 45.39 & 0.338 & 10.39 & 25.24 & & & \\
\hline$G+E L 1 / L 5$ & 0.144 & 67.64 & 97.14 & 0.154 & 28.36 & 82.41 & & & \\
\hline
\end{tabular}

Table 1: Mean ADOP, Percentage of ADOP values below 0.12 cycles and Success Fix solution ratio (FR), for different elevation masks for a GPS-only $(G)$ System or a combined GPS +Galileo $(G+E)$ System.

Figures 6 and7 illustrates the impact of a high elevation mask of $45^{\circ}$ in the positioning performance with a combined GPS+Galileo system in comparative with a GPS-only system. These Figures exhibit the horizontal and vertical error as well as the number of satellites. The mean horizontal and vertical error for a combined GPS + Galileo system are 0.0258 meters and 0.0111 meters respectively. The mean errors are lower in contrast with a GPS-only system with a mean horizontal error of 0.0359 meters and a mean vertical error of 0.0135 meters.

Finally, with the GNSS measurement data acquired in DOY 134 and with an elevation mask of $35^{\circ}$, a dynamical and urban scenario was taken into account as a second validation. An elevation mask of $35^{\circ}$. The difference in the number of fixed solutions of a GPS-only System with respect to a combined GPS+Galileo RTK positioning algorithm approach is depicted in Figure 8.

Figures 9 and 10 illustrate the comparative of fixed solutions between a GPS-only system with respect to a combined GPS +Galileo system. The fixed solutions in this a dynamical scenario are contrasted with a reference trajectory. Figure 8 also exhibits that the number of fixed solutions is superior in a $41.32 \%$ for a combined GPS+Galileo System when a high elevation mask is set with respect to a GPS-only System.

\section{CONCLUSIONS}

With a Galileo System scheduled with a full operational capability in 2020 and with modernized GPS satellites with L5 frequency available, GNSS positioning solutions open the opportunity to the data fusion of multiple satellite systems and also dual-frequency. This study has formulated the benefits under high elevation masks of a dual-frequency combined GPS + Galileo RTK positioning algorithm approach accounting for realistic conditions. The analysis and study of the ADOP for high satellite elevation mask was presented. This study shows that even with a low elevation mask of $10^{\circ}$, a GPS-only System offers a $52.61 \%$ of the mean ASR in comparative with a combined dual-System that offer $99.95 \%$ of the mean ASR. This performance is comparable even with a elevation mask of $45^{\circ}$ when a combined GPS+Galileo System offers a benefit of $57.17 \%$ of the mean ASR with respect to GPS-only System. This efficiency gives an evidence of a strong IAR and by consequence a better position solution approach. This remark is also verified with the percentage of Success fixed solutions where a combined GPS+Galileo-System gives a prominent performance. The validation of this work is significant when environmental constraints are considerable an a high elevation mask is set. 

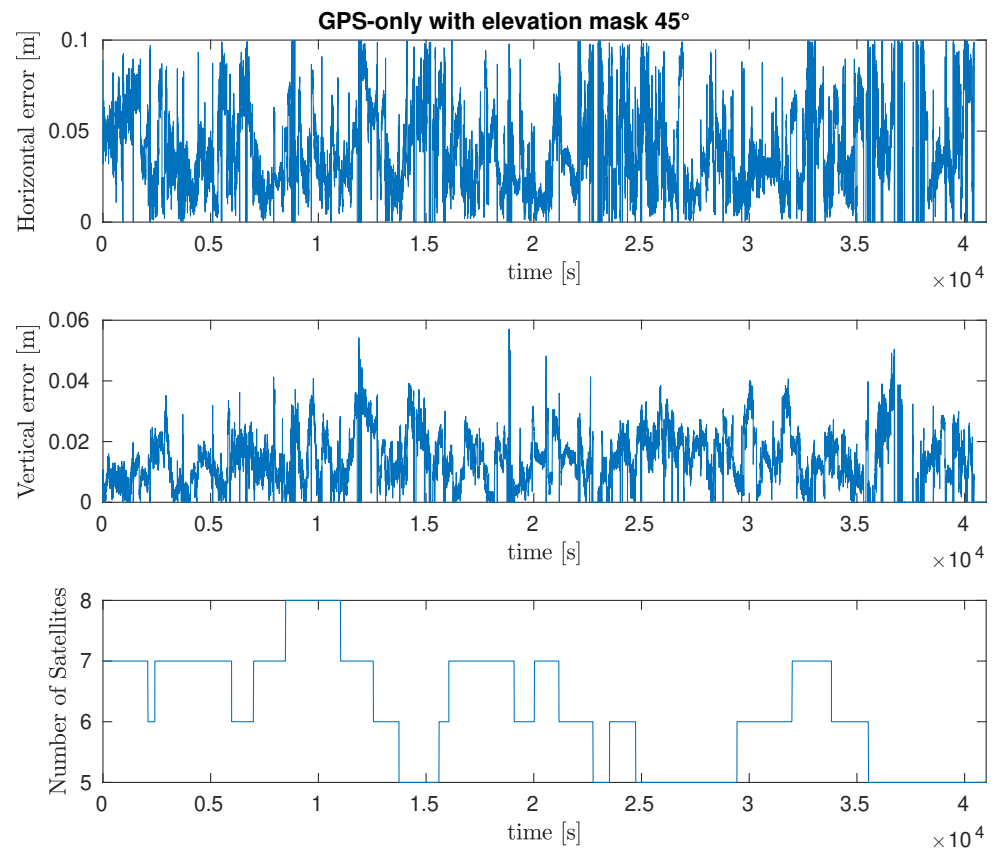

Figure 6: Horizontal and Vertical error for GPS-only RTK positioning solution. Only fixed solutions are considered in this analysis
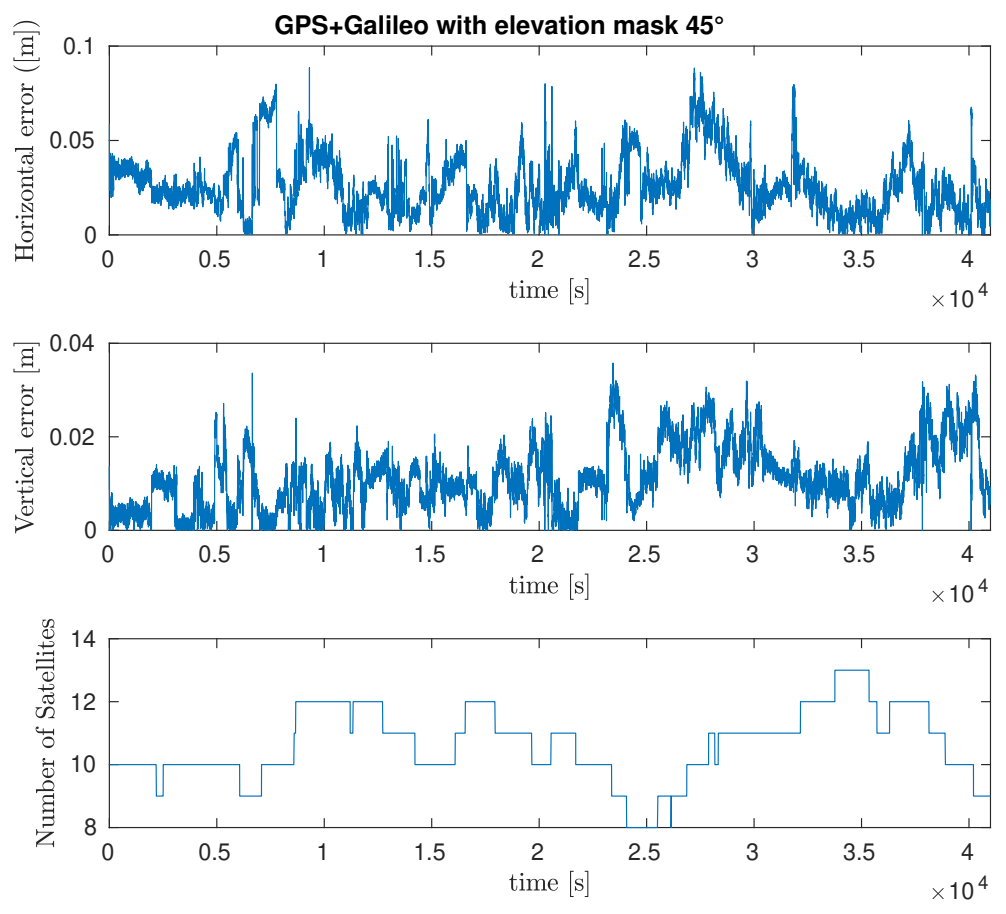

Figure 7: Horizontal and Vertical error for a combined GPS+Galileo RTK positioning solution. Only fixed solutions are considered in this analysis 

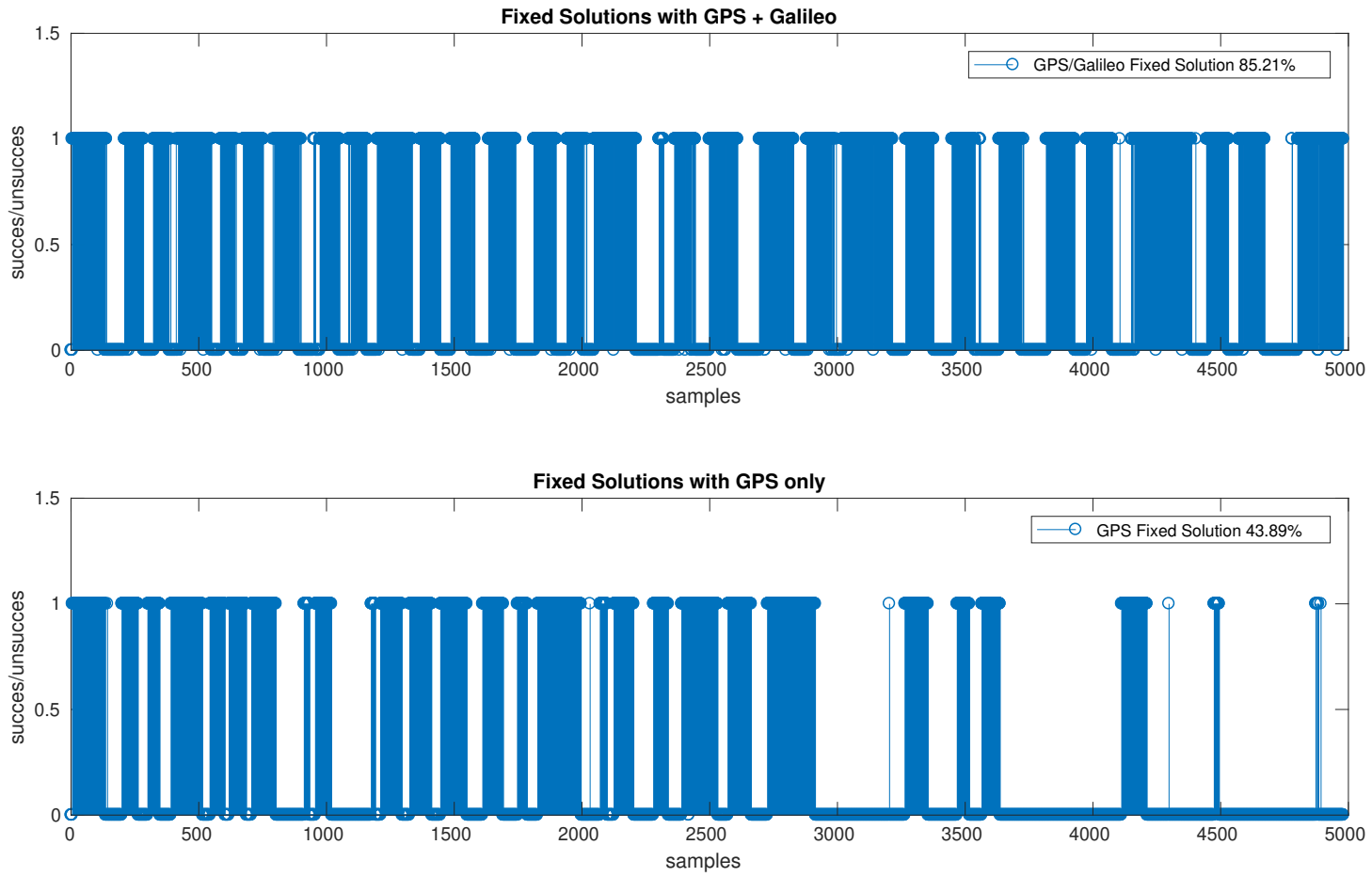

Figure 8: Success and unsuccessful fixed solutions in a dynamical scenario.

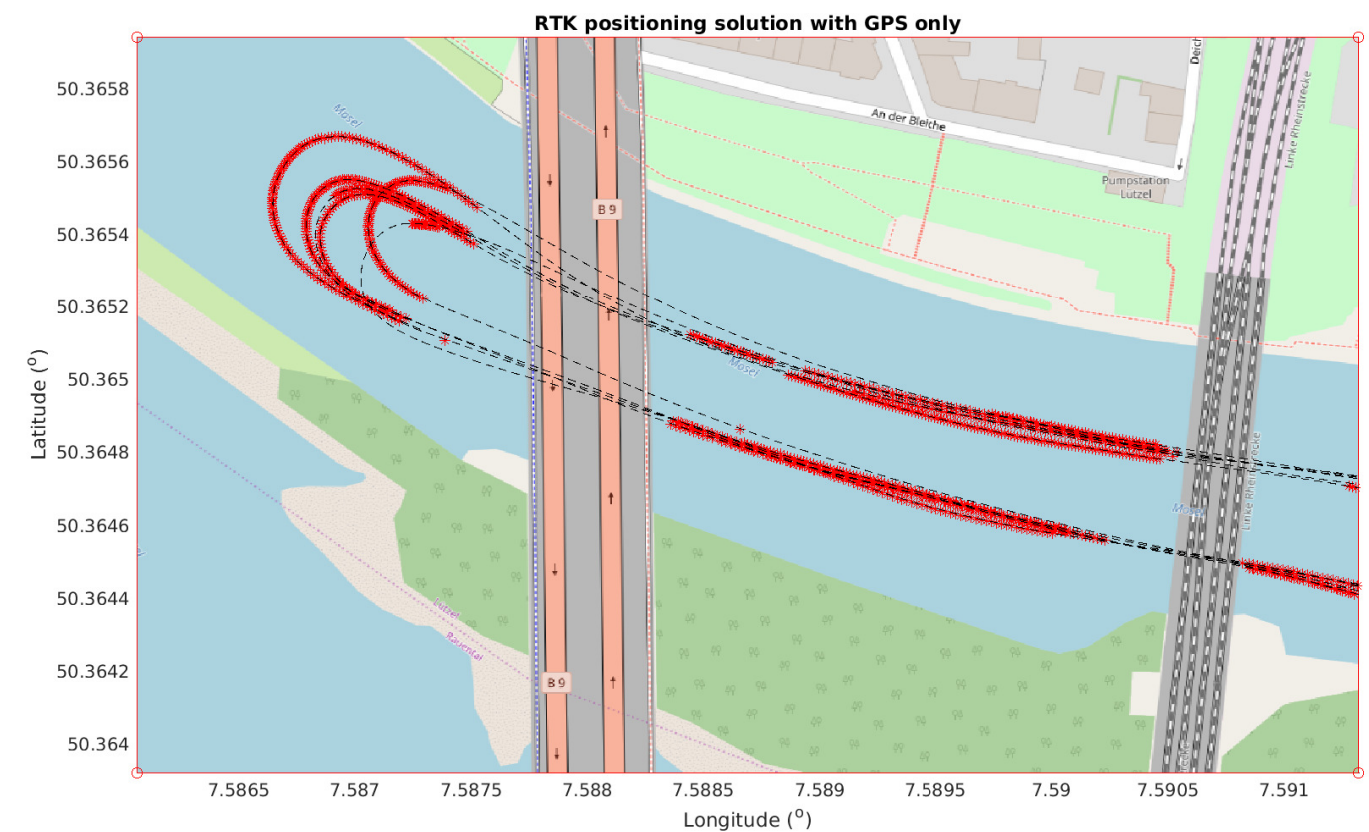

Figure 9: Fixed solutions using a GPS-only RTK positioning algorithm in a dynamical scenario. 


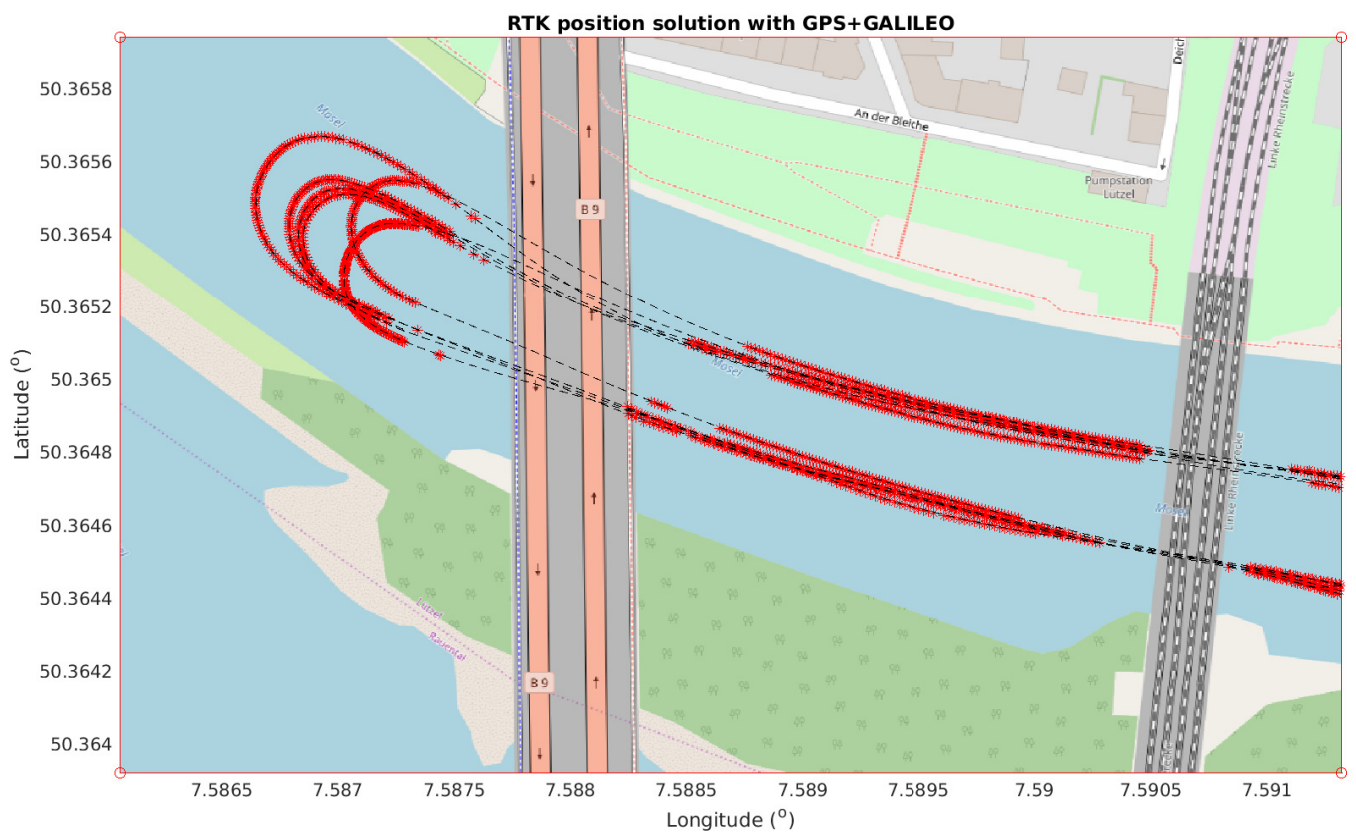

Figure 10: Fixed solutions using a combined GPS+Galileo RTK positioning algorithm in a dynamical scenario.

\section{REFERENCES}

[1] Tuan Li, Hongping Zhang, Xiaoji Niu, and Zhouzheng Gao. Tightly-coupled integration of multi-gnss single-frequency RTK and MEMS-IMU for enhanced positioning performance. Sensors (Switzerland), 17(11), 2017.

[2] Kan Wang, Pei Chen, and Peter J.G. Teunissen. Single-epoch, single-frequency multi-GNSS 15 RTK under high-elevation masking. Sensors (Switzerland), 19(5):1-21, 2019.

[3] Daniel Medina, Anja Heßelbarth, Rauno Büscher, Ralf Ziebold, and Jesús García. On the Kalman filtering formulation for RTK joint positioning and attitude quaternion determination. In 2018 IEEE/ION Position, Location and Navigation Symposium (PLANS), pages 597-604. IEEE, 2018.

[4] Richard B Langley. Rtk Gps. GPS World, pages 70-75, 1998.

[5] D. Montello and A Maglie Larghe. Least-Squares Estimation of the Integer GPS Ambiguities. (August), 1993.

[6] P. J. G. Teunissen, P. J. de Jonge, and Christian C. C. J. M. Tiberius. The LAMBDA-Method for Fast GPS Surveying. Proceedings of International Symposium «GPS technology applications»', (1):203-210, 1995.

[7] P. J.G. Teunissen, R. Odolinski, and D. Odijk. Instantaneous BeiDou+GPS RTK positioning with high cut-off elevation angles. Journal of Geodesy, 2014.

[8] PJG Teunissen, Robert Odolinski, and Dennis Odijk. Instantaneous BeiDou+ GPS RTK positioning with high cut-off elevation angles. Journal of geodesy, 88(4):335-350, 2014.

[9] Robert Odolinski, Peter JG Teunissen, and Dennis Odijk. Combined BDS, Galileo, QZSS and GPS single-frequency RTK. GPS solutions, 19(1):151-163, 2015.

[10] P. J.G. Teunissen. A canonical theory for short GPS baselines. Part IV: Precision versus reliability. Journal of Geodesy, 1997.

[11] D. Odijk and P. J G Teunissen. ADOP in closed form for a hierarchy of multi-frequency single-baseline GNSS models. Journal of Geodesy, 82(8):473-492, 2008.

[12] Peter J G Teunissen and Dennis Odijk. Ambiguity dilution of precision: Definition, properties and application. Proceedings of ION GPS, 1(October):891-899, 1997.

[13] Bruno M Scherzinger, Applanix Corporation, and Richmond Hill. Precise Robust Positioning with Inertial / GPS RTK. System, pages 155-162, 1983.

[14] Björn Reuper, Matthias Becker, and Stefan Leinen. Benefits of multi-constellation/multi-frequency GNSS in a tightly coupled GNSS/IMU/Odometry integration algorithm. Sensors (Switzerland), 18(9):1-25, 2018.

[15] Wang Gao, Xiaolin Meng, Chengfa Gao, Shuguo Pan, and Denghui Wang. Combined GPS and BDS for single-frequency continuous 
RTK positioning through real-time estimation of differential inter-system biases. GPS Solutions, 22(1):1-13, 2018.

[16] Daniel Medina, Vincenzo Centrone, Ralf Ziebold, and Jesús García. Attitude Determination via GNSS Carrier Phase and Inertial Aiding. In ION GNSS+, The International Technical Meeting of the Satellite Division of The Institute of Navigation, Miami, FL, Sep 2019.

[17] R Odolinski, Peter Teunissen, and Dennis Odijk. An analysis of combined COMPASS/BeiDou-2 and GPS single-and multiplefrequency RTK positioning. In Proceedings of the ION 2013 Pacific PNT Meeting, pages 69-90. INST NAVIGATION, 2013.

[18] Daniel Medina, Kasia Gibson, Ralf Ziebold, and Pau Closas. Determination of Pseudorange Error Models and Multipath Characterization under Signal-Degraded Scenarios. In PROCEEDINGS OF ION GNSS+. Institute of Navigation, 2018.

[19] Peter JG Teunissen and Sandra Verhagen. The GNSS ambiguity ratio-test revisited: a better way of using it. Survey Review, 41(312):138-151, 2009.

[20] Sandra Verhagen and Peter JG Teunissen. The ratio test for future GNSS ambiguity resolution. GPS solutions, 17(4):535-548, 2013.

[21] Ying Xu and Wu Chen. Performance analysis of GPS/BDS dual/triple-frequency network RTK in urban areas: A case study in Hong Kong. Sensors (Switzerland), 18(8), 2018.

[22] Daniel Arias Medina, Michailas Romanovas, Iván Herrera-Pinzón, and Ralf Ziebold. Robust position and velocity estimation methods in integrated navigation systems for inland water applications. In 2016 IEEE/ION Position, Location and Navigation Symposium (PLANS), pages 491-501. IEEE, 2016. 